\title{
Defining Bacterial Regulons Using ChIP-seq
}

\section{Methods}

Kevin S. Myers, Dan M. Park, Nicole A. Beauchene, Patricia J. Kiley

Kevin S. Myers:

Laboratory of Genetics, University of Wisconsin - Madison; Madison, WI; USA; 53706

Great Lakes Bioenergy Research Center; University of Wisconsin - Madison; Madison, WI; USA; 53706

Dan M. Park:

Lawrence Livermore National Laboratory, Livermore, California, USA

Nicole A. Beauchene:

Department of Biomolecular Chemistry; University of Wisconsin - Madison; Madison, WI; USA; 53706

Patricia J. Kiley

Department of Biomolecular Chemistry; University of Wisconsin - Madison; Madison, WI; USA; 53706

Great Lakes Bioenergy Research Center; University of Wisconsin - Madison; Madison, WI; USA; 53706

Correspondence to: Patricia J. Kiley; Department of Biomolecular Chemistry, 4204C Biochemical Sciences Building, University of Wisconsin, 440 Henry Mall, Madison, WI 53706; Tel: +1 (608) 262-6632; Fax: +1 (608) 262-5253; Email: pjkiley@ wisc.edu 
1 Abstract

2

3 Chromatin immunoprecitation followed by high-throughput sequencing (ChIP-seq) is a powerful

4 method that identifies protein-DNA binding sites in vivo. Recent studies have illustrated the

5 value of ChIP-seq in studying transcription factor binding in various bacterial species under a

6 variety of growth conditions. These results show that in addition to identifying binding sites,

7 correlation of ChIP-seq data with expression data can reveal important information about

8 bacterial regulons and regulatory networks. In this chapter, we provide an overview of the

9 current state of knowledge about ChIP-seq methodology in bacteria, from sample preparation to

10 raw data analysis. We also describe visualization and various bioinformatic analyses of

11 processed ChIP-seq data.

12 


\section{Section 1: Introduction}

Transcriptional regulation of gene expression by transcription factors (TFs) is a common

16 mechanism of regulation in bacteria [1]. Identifying all the genes directly regulated by a

17 transcription factor can be challenging particularly for those that regulate a large number of

18 genes and whose DNA binding sites might be less conserved and thus difficult to identify using

19 bioinformatic approaches. By comparing RNA profiles of strains lacking the transcription factor

20 to their parent strain, using either microarray technology or high-throughput sequencing

21 technology, candidate genes controlled by a transcription factor can be identified. However,

22 whole-genome expression analyses cannot reveal whether the influence of the TF on RNA levels

23 is direct or indirect. This requires identification of TF binding within the appropriate promoter

24 region. While it is possible to characterize binding to these regions using in vitro assays, recent

25 advances in genome wide analysis, make identification of these binding sites possible in vivo,

26 allowing identification of binding events under the same growth conditions that RNA levels were

27 determined.

Chromatin immunoprecipitation (ChIP) followed by microarray analysis (ChIP-chip) or,

29 more recently, high-throughput sequencing (ChIP-seq), which has a much higher resolution and

30 signal-to-noise ratio than ChIP-chip, has been used to map genome-wide binding of many

31 bacterial TFs [2-25]. While many of the studies have focused on TFs in Escherichia coli, recent

32 studies have examined transcription factors in many diverse bacterial species, such as Vibrio

33 cholerae, Vibrio harveyi, Rhodobacter sphaeroides, Salmonella enterica, Mycobacterium

34 tuberculosis, and Caulobacter crescentus [2-25]. A subset of these studies also correlated

35 occupancy data with expression data to investigate the regulons of certain TFs

$36[2,3,5,7,8,13,16,21,22,25]$, and we predict the desire to use these assays to study other TFs will 
37 increase as the costs of the performing ChIP-seq decrease. Here, we provide an overview to

38 using ChIP-seq to identify bacterial regulons, from sample preparation, data generation, data

39 visualization, data analysis, and downstream bioinformatic and computational analyses.

40 We offer a general overview of each step and highlight specific analyses that have aided

41 our work. It is important to note that analysis of ChIP-seq data in bacteria is an evolving pipeline

42 and as such, new approaches and algorithms are being introduced frequently. It is beyond the

43 scope of this review to provide a comprehensive description of known variations of ChIP-seq

44 analysis, including the higher resolution variant, ChIP-exo. ChIP-exo has shown promise in the

45 few bacterial studies where it has been used [26-29] but the analysis of ChIP-exo data has its

46 own unique issues including sample preparation and data analysis. We encourage those

47 interested in performing ChIP-seq experiments or variations on ChIP-seq to review other studies

48 [2-25] if they want a broader sense of the experimental and analytical variables.

\section{Section 2: ChIP-seq Sample Preparation and Data Generation}

\section{$50 \quad 2.1$ General Overview of ChIP-seq Procedure}

51 ChIP-seq reports on the DNA sequences that can be cross-linked by formaldehyde to a

52 given transcription factor in actively growing cells and then enriched relative to genomic DNA

53 when the DNA-protein complexes are precipitated by use of an antibody specific to the

54 transcription factor. Aspects of the ChIP-seq method and resulting data analysis will be described

55 in detail throughout this review (Figure 1). Several methods and review articles describe in detail

56 how to perform ChIP-chip and ChIP-seq experiments [30-33], and we encourage readers to also

57 review these publications. The methodology described in these articles differs and while each has

58 been used to produced high-quality ChIP-seq data, we have found success following the method

59 described by Davis et al [33]. Briefly, cells are grown in desired conditions to a designated 
60 growth phase and formaldehyde is added to crosslink proteins to DNA. Cells are pelleted, lysed,

61 the lysate is sonicated to shear the DNA, and DNA-TF complexes of interest are enriched using

62 an antibody specific to the TF being studied. The crosslinks are reversed by heating the sample

63 to $65^{\circ} \mathrm{C}$ and the DNA that was bound to the TF of interest, the immunoprecipatant (IP), is

64 isolated and sequenced along with a sample of DNA from un-enriched, non-antibody treated

65 DNA (Input sample). The Input sample represents the background signal of available chromatin

66 for IP, controlling for regions of genomic DNA that may be enriched for reasons other than TF

67 binding. Areas of the genome bound by the TF are recovered in larger proportion in the IP

68 fraction than unbound areas. Thus, areas of TF binding are regions of the genome that show

69 enrichment in the IP fraction compared to the background Input fraction (Figure 1). In the next

70 sections, we discuss what we consider "best practices" in designing ChIP-seq experiments.

\subsection{Selection of TF and Experiment Design}

72 The design of a ChIP-seq experiment for regulon analysis begins with the selection of the

73 TF and designing a strategy for its expression. The use of transcription factor-specific antibodies

74 is critical to the success of ChIP-seq experiments. The two primary methods to study TF binding

75 involve using antibodies specific for the native protein or antibodies to a "tag" contained within a

76 genetically engineered tagged variant of the TF. Both types of antibodies have been used to

77 produce high quality ChIP-seq data and the choice depends on time, cost consideration,

78 functionality of the tagged transcription factor and genetic tractability of the organism studied.

79 Since commercial antibodies are available for only a small subset of native bacterial TFs,

80 it is necessary to raise antibodies against the TF of interest, which requires additional time and

81 expenses associated with protein purification and antibody production. It is critical to test the

82 specificity of the antiserum by performing western blot analysis using cell lysates prepared from 
83 wild-type and from a strain lacking the TF of interest. If cross-reactivity to other proteins is

84 detected, additional purification of the antibody should be performed using affinity

85 chromatography with the purified TF of interest immobilized to a column or by absorbing the

86 antiserum with an acetone powder prepared from a strain lacking the protein of interest. Both

87 methodologies have been successfully utilized in our laboratory to yield highly specific antibody

88 solutions $[2,3]$.

89 It is possible that antibodies recognizing the native protein are not specific enough for

90 ChIP, even after purification. Many successful ChIP-seq studies have used affinity-tagged

91 versions of the TF of interest, often using triple FLAG affinity tags [4,5,7,8,12,15,18,21-25] or

92 myc tags [14]. Using an affinity-tagged protein allows the use of a commercial antibody that

93 likely has little cross-reactivity with other proteins from the bacterium under study and negates

94 the requirement of an antibody specific to the TF of interest. However, the addition of a tag to

95 the TF may affect its ability to bind DNA, interact with RNA Polymerase (RNAP), or be

96 regulated by another factor (e.g. protein or small molecule). Thus, use of tagged proteins requires

97 additional in vitro and in vivo control experiments to ensure the activity of a tagged protein is

98 similar to that of the native protein, such as comparing expression and DNA binding of tagged

99 TFs to native TFs. However, another requirement of using a tagged version of the TF is that the

100 method requires the ability to introduce a stable plasmid encoding the tagged transcription factor

101 or to edit the genome to replace the wild-type transcription factor with the tagged variant. While

102 this is relatively simple for some bacteria, other species lack genetically tractable systems.

103 Therefore, for these bacteria, the only option is to raise antibodies against the TF of interest.

104 Finally, if the TF of interest is present at a low cellular copy number or if the

105 environmental condition that activates a TF is unknown, it may be necessary to over-express the 
106 TF in order to detect a ChIP-signal. For example, many $\sigma$ factors must be released from anti-

107 sigma factors for activity [1]. If the environmental signal that causes release of the $\sigma$ factor is

108 unknown, over-expression of the $\sigma$ factor may allow for detection of binding sites with ChIP-

109 seq. This method of TF overexpression was used to map TF binding sites in Mycobacterium

110 tuberculosis because the environmental signal required for activation was unknown [22]. Such

111 over-expression can be accomplished by engineering a plasmid where the TF gene is expressed

112 from an inducible promoter. However, over-expression may also result in binding to weaker

113 affinity sites on the genome that may not be bound at wild-type concentrations of the TF.

114 Therefore, care must be taken to determine which sites are bound under physiologically relevant

115 conditions and to show that they are dependent on the inducing signal.

\section{$116 \quad 2.3$ Checking the ChIP Procedure}

117 In this section, we highlight specific steps that we have found important for successful

118 ChIP-seq experiments. The method used for immunoprecipitation of the cross-linked DNA

119 bound transcription was described by Davis et al [33]. Since library preparation and sequencing

120 are still expensive and can take weeks to obtain data depending on access to sequencing cores,

121 pilot experiments to examine the efficiency of ChIP before subjecting the samples to ChIP-seq

122 are critical. The amount of antibody to add to cultures and the duration of antibody treatment will

123 vary depending on the affinity of the antibody to the protein of interest and should be optimized

124 empirically. ChIP-qPCR, wherein the isolated IP and Input DNA amount is measured using

125 qPCR can be performed on samples where the amount and incubation time of the antibody are

126 varied to evaluate optimal conditions for enrichment. If targets of the TF are known, amplifying

127 targets of the TF of interest, including high-affinity targets, moderate-affinity targets, and low-

128 affinity targets as well as regions of the genome not bound by the TF of interest should provide 
129 sufficient information to optimize conditions. By comparing the enrichment by qPCR of target

130 sites to those not bound by the TF, the efficacy and efficiency of the ChIP experiment can be

131 evaluated [33]. However, if the TF being studied lacks known target sites, one approach is to use

132 the promoter region of the TF gene, since autoregulation is a common feature of bacterial TFs

133 [34]. Many potential targets may need to be evaluated to obtain reliable ChIP-qPCR results.

134 Several controls are useful in curating the list of TF binding regions. First, the Input DNA

135 is used in all ChIP-seq experiments to measure the background enrichment to eliminate

136 nonspecific binding regions. Second, comparing the enrichment signal in a control strain lacking

137 the TF or containing an untagged version of the TF is used to reveal any non-specific enrichment

138 due to aberrant binding of the antibody, reducing the downstream analyses of false positive areas

139 of enrichment. Alternatively, if a mutant strain is not available, it might be possible to perform

140 ChIP-seq under growth conditions in which the protein of interest is inactive and shows no

141 binding activity. However, the results of this approach should be interpreted with caution as the

142 inactive TF may still exhibit residual DNA binding. Comparison of TF binding sites between

143 different growth conditions is discussed in more detail in Section 3.3.

144 The ideal number of biological replicates for ChIP-seq experiments will depend on the

145 desired downstream analyses. High quality and reproducible data using two biological replicates

146 has shown to be sufficient for identification of highly enriched DNA binding locations $[2,3]$.

147 However, at least three biological replicates will aid in identifying less enriched regions and also

148 will provide greater statistical power for more robust analyses, such as differential binding

149 analyses [35,36].

$150 \quad$ 2.4 Sequencing ChIP DNA Samples 
152 fragmented and size selected and sequencing adapters are ligated to the DNA fragments - and

153 they are sequenced using a high-throughput sequencing platform, which is often accomplished at

154 a sequencing facility. We have had success following the Illumina's guidelines for library

155 preparation, which is time intensive, involving many steps and reagents. A step-by-step protocol

156 for ChIP-seq library construction is also described in a recent review [30]. Nevertheless, some

157 samples may require additional troubleshooting during library preparation. Although our

158 sequencing data have been generated using the Illumina technology, much of the procedure for

159 analysis of sequencing results is independent of the technology. Illumina sequencing can be

160 either single-end (sequenced from one end of the DNA fragment) or paired-end (sequenced from

161 both ends of the DNA fragment) [37]. Successful ChIP-seq experiments have been performed

162 using both methodologies. Furthermore, multiple biological samples can be sequenced together -

163 multiplexed - by using unique DNA sequences, termed barcodes or indexes, when constructing

164 the sequencing library [37]. These unique barcodes or indexes allow for the identification of the

165 sequence data from each sample present in the sequencing experiment. Additionally,

166 multiplexing can decrease the cost of the sequencing experiment, allowing additional biological

167 replicates to be obtained. However, if multiplexing, total sequencing depth must be high enough

168 to ensure enough reads for every experiment for proper analysis (at least 2-3 million reads per

169 sample).

$170 \quad 2.5$ Processing Sequencing Results

171 The process of analyzing ChIP-seq data is currently an evolving pipeline and, as such,

172 there is not a single best way to evaluate the data. We provide some examples here and in the

173 following sections, but also encourage researchers to fully evaluate each algorithm used to 
174 determine if settings should be changed to match your particular experimental design.

175 Furthermore, ChIP-seq data is publically available in NCBI GEO

176 (http://www.ncbi.nlm.nih.gov/geo/) and EMBL-EBI (http://www.ebi.ac.uk/), making it easy for

177 all researchers to re-analyze data if potentially better analysis pipelines become available.

178 The first step in analysis of ChIP-seq data is to evaluate the quantity and quality of the

179 ChIP-seq sequencing reads produced from the sequencing instrument. The number of reads in

180 each experiment reflects the levels of coverage; downstream analyses may need to be normalized

181 for any differences in total read number. For each read, the quality is measured as confidence of

182 the base identity (quality score) at each position, which, for Illumina sequencing data, is reported

183 in a FASTQC file associated with each experiment (Table 1). A low quality score for reads can

184 affect the efficiency of alignment and thus the quality of the ChIP-seq data [37]. Generally, the

185 quality scores within reads tends to decrease at both ends of the read. This initial examination is

186 important to determine the quality and quantity of the raw data from the sequencing experiment

187 before processing the data for biological analysis.

188 There are many tools and algorithms that can be used to process ChIP-seq data for

189 biological analysis, and a full description and evaluation of each is beyond the scope of this

190 review. We have utilized an on-line suite of tools called Galaxy [38]. The Galaxy system

191 (www.usegalaxy.org) [38] is an open-source, user-friendly, web-based platform for data

192 intensive biological research of next-generation sequencing data built from commonly used

193 algorithms and tools. It also records the processes performed at each step, allowing full recall of

194 every step performed in analysis. Many of the software packages present in Galaxy, along with

195 many additional software packages, can be downloaded and run by the user individually, often

196 on the command line. We will refer to these throughout this and the next sections. Regardless of 
197 the methods used, it is very important that careful records be kept on the analysis of ChIP-seq

198 data. This includes the version of tools used as well as any changes made to the default settings

199 for each algorithm. These records will allow future researchers to fully understand and, if

200 necessary, repeat your analyses.

201 The first step is to process the sequencing reads to remove bases of low quality. This is

202 accomplished by selectively measuring the quality of each base read and removing (trimming)

203 bases from the reads based on read quality [39]. When trimming, it is important to ensure that

204 reads are of a minimum length to ensure unambiguous alignment. After trimming, the

205 sequencing reads are aligned to a reference genome to identify the genomic location of

206 enrichment. A high-quality reference genome is required for optimal alignment, and many

207 reference genomes can be obtained from NCBI (http://www.ncbi.nlm.nih.gov/genome/). Two

208 common alignment algorithms are Bowtie2 [40] and BWA [41]. Both algorithms output the

209 alignment data as a SAM file and/or a binary version of a SAM file called a BAM file (Table 1)

210 [42]. Regardless of the algorithm used, the primary goal is to align as many of the sequence

211 reads as possible to the reference genome as accurately as possible to ensure reliable downstream

212 analysis.

213 Section 3: Identifying Areas of Enrichment in ChIP-seq Experiments

214 After sequencing reads are aligned to the genome, there are many analyses that can be

215 performed. In the following sections, we describe examples of these analyses that have proven

216 useful in studying bacterial regulons. However, it is important to note that while many of these

217 analyses are common to all ChIP-seq experiments (e.g. peak calling), some analyses are required

218 for specific TFs or biological questions.

2193.1 Use of Peak Calling Algorithms 
After the sequencing reads are aligned to the genome, the next step is to identify areas of

221 enrichment in the ChIP-seq data that represent where the TF binds throughout the genome,

222 referred to as peaks. The robust identification of peaks is crucial for the success of a ChIP-seq

223 analysis. Peak finding algorithms are intended to identify areas of enrichment in ChIP-seq data.

224 These algorithms are important because they remove some subjectiveness from visual peak

225 calling and provide a statistical basis for determining areas of enrichment. Examples of

226 algorithms that have been used for bacterial ChIP-seq analysis include CisGenome [43], MACS

227 [44], and MOSAiCS [45]. We have used MOSAiCS, which, unlike many other peak calling

228 algorithms, was designed to analyze ChIP-seq data from bacteria as well as eukaryotes.

229 MOSAiCS can also identify areas of enrichment for DNA binding proteins that display broad

230 regions of enrichment, such as RNAP or nucleoid-like proteins like H-NS [45] rather than just

231 symmetrically shaped peaks. However, it may be useful to analyze the ChIP-seq data with

232 multiple algorithms and compare the enriched regions identified. While this would increase the

233 computational and analysis time for your experiment, it would also provide multiple lines of

234 evidence for enrichment at a particular genomic region.

235 It is important to note that as with any algorithm, the results provided by these tools may

236 not include all possible peaks or could contain false positives. Therefore, care must be taken to

237 ensure proper optimization of the algorithm for your experiment. For example, adjusting the

238 false discovery rate (FDR) of a peak-calling algorithm will affect the number of peaks identified

239 - a lower FDR will decrease the number of both total peaks identified as well as false-positive

240 peaks identified. Furthermore, peak calling algorithms simply identify whether an area of the

241 genome is enriched or not based on a statistical threshold - a binary identification. Thus, many

242 peak calling algorithms may miss peaks with low enrichment above background. Additional 
243 visual inspection may be required to identify high value peaks in a ChIP-seq dataset, especially

244 peaks with low enrichment (Section 4). The identification of peaks of low enrichment remains a

245 weak aspect of ChIP-seq analysis and hopefully new algorithms will be developed that improve

246 such peak calls.

247 3.2 Deconvolution of ChIP-seq Peaks

248 Some bacterial TFs bind to multiple, closely spaced locations within a promoter region

249 (less than 100 bp separation) [46]. The ability to distinguish between these sites yields greater

250 mechanistic insight into the transcriptional control mediated by the TF of interest. However,

251 most standard peak finding algorithms are unable to resolve closely spaced binding sites, instead

252 misidentifying these regions as a single binding site with the wrong genomic coordinates. To test

253 for closely spaced binding sites, peak deconvolution algorithms such as CSDeconv [20,47],

254 GEM [48], PICS [49] or dPeak [46] can be used. We found that dPeak was able to identify

255 regions containing multiple TF binding sites, most of which were missed with standard peak

256 finding algorithms [46]. Furthermore, dPeak was designed to leverage the greater resolution

257 provided by paired-end sequencing data to provide high-resolution ChIP-seq binding site

258 locations in bacteria [46]. The advantage of paired-end reads for distinguishing closely spaced

259 binding sites stems from knowing the exact length of each read, allowing a single read to be

260 unequivocally associated with one or both of two closely spaced binding sites. In contrast, for

261 single-end sequencing, a single read cannot be assigned with certainty to one or both binding

262 sites since the 3' end must be approximated, typically by extending by the average library size.

263 Thus, reads spanning only one binding event may be wrongly extended to cover both binding

264 sites while long reads spanning both sites may not be under extended and cover only one binding

265 site, leading to reduced sensitivity [46]. Therefore, if it is suspected that the protein of interest 
266 may have multiple binding sites located close together, then paired-end ChIP-seq data should be

267 obtained to maximize the advantages of the dPeak algorithm.

It may be of interest to compare ChIP-seq data between growth conditions to study how

270 different environments affect binding site occupancy or to compare wild type to the control

271 lacking the TF of interest. When comparing biological replicates from different growth

272 conditions, the differences in total read number must be normalized across samples. However,

273 the best strategy for normalization is yet to be determined. Normalization to the background in a

274 ChIP-seq experiment can be performed locally (surrounding only identified peaks) or on a global

275 scale (normalize to all un-enriched genomic regions) [2]. Alternatively, spiking the DNA sample

276 with synthetic DNA fragments will provide external reference sequences of known amounts for

277 normalization [50].

278 After normalization, samples can then be compared for differential binding by the

279 transcription factor. Due to the symmetrical, peak shape of enriched regions from most TFs,

280 differential binding can be assessed by comparing the number of sequencing reads aligned

281 around the peak summit. When comparing binding of other proteins, such as H-NS, which shows

282 long regions of binding across the genome, it is better to compare the sequencing coverage under

283 the entire enriched region [2]. In our studies, we have used the algorithm DBChIP [35], which

284 was developed specifically to compare ChIP-seq enrichment of TF peaks between two

285 experimental conditions, with multiple biological replicates for each condition. For example, we

286 successfully used DBChip to identify oxygen-dependent differences in $\sigma^{70}$ binding by analyzing

$287 \quad \sigma^{70}$ ChIP-seq signal in E. coli under aerobic and anaerobic growth conditions (Figure 2) [2]. It is

288 important to note that differential binding analysis is appropriate when comparing enrichment of 
289 the same site under different conditions. In contrast, we have found that ChIP-seq enrichment

290 does not necessarily correlate with binding strength [2], thus preventing comparison of sites

291 within the genome. Many factors likely determine the intensity of peaks across genomic sites,

292 such as inherent cross-linking efficiency within a region of DNA [2]. We assume that these

293 factors should remain constant across conditions.

\section{Section 4: Visualization of ChIP-seq Data}

\section{4.1 Importance of Visually Evaluating ChIP-seq Data}

296 We have found that visual inspection is an important part of analyzing ChIP-seq data.

297 Visual inspection is generally required to identify peaks with low enrichment above background,

298 which current peak calling algorithms are unable to identify without introducing many false

299 positives. Such low enrichment may be due to poor cross-linking efficiency or low sequencing

300 coverage at that location in the genome. However, visual analysis of ChIP-seq data lacks

301 statistical support and thus additional peaks identified only visually should be subjected to

302 additional validation, which is discussed in Section 7.3. Visualization can also be useful in

303 identifying false positive peaks. Canonical TF binding sites yield a bimodal enrichment profile

304 where enrichment on the forward and reverse strands are staggered as a result of sequencing the

$3055^{\prime}$ portion of each DNA fragment [51,52]. Artifactual peaks typically lack this staggered peak

306 signature exhibiting reads specific to only one strand, and thus often can be readily identified

307 through visualization of reads mapping to both the forward and reverse strand. Algorithms such

308 as QuEST [51] compares the enrichment profiles of both strands and can be used to filter out

309 such artifactual peaks. Visual inspection of the data may also elucidate other trends that may

310 have biological significance such as long binding regions, which might be overlooked when

311 using the algorithmic results alone. Finally, visually evaluating ChIP-seq data can be important 
312 when considering the physiological context of binding events. The best way to visually inspect

313 the data is to plot the ChIP-seq data on a genome browser and step through the genome.

314 4.2 Preparing Files for Visualization

315 The most common file format used for visualization of ChIP-seq data is the wiggle (or

316 WIG) file (Table 1). Wiggle files are designed to display information dense data, such as ChIP-

317 seq data throughout the genome. These files are generated by enumerating how often each read

318 corresponds to each base in the genome. A composite wig file that incorporates data from both

319 the forward and reverse strands is typically generated for visualization of ChIP-seq data;

320 however, wig files for both the forward and reverse strand are useful for visualizing the

321 staggered peak profiles described above. To generate composite wig files, we use the software

322 package QuEST [51], that applies a peak shift estimation to generate a composite density profile

323 for each enriched region. In addition to the wiggle file, visualization depends on an annotated

324 genome. The more robust the annotation of the genome file, the more useful it will be for

325 visualization analysis.

3264.3 Visualization Using MochiView

327 There are many genome browsers available that can accept wiggle files and visualize

328 ChIP-seq data. We use the genome browser MochiView [53] (Figure 2). MochiView was

329 developed for use with yeast data, but functions very well to visualize multiple data types,

330 including ChIP-seq, on a bacterial genome. MochiView is a fast browser that can accept many

331 tracks of data without affecting performance. It is also highly customizable, allowing for easy

332 generation of publication ready figures. All data are stored in a database in MochiView, which

333 can be exported and shared, allowing easy transfer of data with other researchers. 
335 data, transcriptomic data, proteomic data, motif location data, and any other gene centric data set,

336 allowing for simple comparison between multiple data types. It can also import the results of

337 peak finding algorithms, deconvolution algorithms, and differential binding analysis. Finally,

338 MochiView is written in Java, and therefore is operating system agnostic and runs equally well

339 in Windows and Mac OS environments. One drawback of MochiView is that it is installed and

340 run locally on your computer. So while you can easily share databases among users, MochiView

341 does not lend itself to visualization via a web browser or server based method, where many users

342 simultaneously use the same database. However, this drawback aside, MochiView has proven a

343 robust and powerful genome browser.

\section{$344 \quad 4.4$ Visualization Online}

345 While local visualization with MochiView is our preferred way of analyzing ChIP-seq

346 data, it is often advantageous to have a shared browser that anyone with a web browser can

347 access. A commonly used genome browser is the UCSC Genome Browser

348 (http://genome.ucsc.edu/index.html) [54]. This is built on the GBrowse genome browser [55],

349 but has many tracks of annotation pre-loaded, making it easy to visualize ChIP-seq data quickly.

350 Additionally, published tracks are available to be loaded into the browser, providing a platform

351 to compare between different experiments from different research groups.

352 It is also possible to download and install an instance of GBrowse or another popular

353 browser JBrowse [56] on a local server for which access can be controlled [55]. Tracks can be

354 downloaded easily from UCSC for use in a personal instance of GBrowse. This can allow many

355 users to access the same data and add to the data in a central location.

\section{Section 5: Associating ChIP-seq Peaks with Genes}


After binding regions are identified, the next step in ChIP-seq analysis is to associate the

358 peaks with the corresponding genes. Ideally, the peak could be algorithmically associated with

359 genes based on proximity of the peak to the transcription start site (TSS). However, many genes

360 in E. coli, the organism that we study, lack known transcription start sites, even with recent

361 studies that have globally identified TSSs [57,58]. The translation start site, which is predicted

362 for all known E. coli protein coding genes, can also be used in such an automated algorithm, but

363 this may produce many false associations because the distance from the translation start site and

364 the TSS is variable. Furthermore, such an automated analysis may ignore transcription start sites

365 that are either far upstream of the translation start site or are located within genes or encode

366 small RNAs.

367 We use visual inspection of each area of enrichment to determine what is the nearest

368 operon as the most accurate way to ensure each peak is best associated with the corresponding

369 operon. Such visual association also ensures that divergent promoters are identified and that sites

370 located within genes are not overlooked. However, for a peak located within a region with

371 divergently transcribed genes, it is impossible to associate the peak with either operon using

372 ChIP-seq data alone, but the use of expression data can suggest which gene is regulated by the

373 TF (Section 7). This will also potentially identify ChIP-seq peaks that do not associate with

374 annotated genes, but may associate with known or unknown small RNAs [2].

\section{Section 6: Motif Analysis Using ChIP-seq Data}

376 6.1 Value of ChIP-seq Data in Identification of Motifs

377 Since ChIP-seq data provides high-resolution ( 10 bp [37]), location information of TF

378 binding sites, the sequences bound by the TF can be analyzed to identify over-represented

379 sequences. Many of motifs present in the literature have been generated from a small number of 
380 binding sites. The use of ChIP-seq data provides dozens to hundreds of binding site sequences,

381 improving the power of motif identification. Updated motifs can aid in identifying false negative

382 binding regions of the genome and can be used for further downstream biochemical and genetic

383 experiments to examine binding efficiency [2].

$384 \quad 6.2$ Motif Identification from ChIP-seq Data

385 Many algorithms have been developed to search a given set of sequences and identify

386 over-represented motifs. Regardless of the algorithm used, all require the sequences extracted

387 from areas of enrichment. These areas may be the entire area of enrichment identified by

388 MOSAiCS or specific genomic coordinates from a deconvoluted peak. Once the DNA sequences

389 are obtained, they are submitted to an algorithm to search for a motif [59]. A commonly used

390 algorithm is MEME [60] - or the related MEME-chip, which is designed for larger sequence

391 inputs [61] - which can be run either online or locally via the command line [62]. In addition to

392 MEME, other motif identification algorithms include info-gibbs [63] and consensus [64] which

393 can be easily accessed at the Regulatory Sequence Analysis Tools (RSAT, http://rsat.ulb.ac.be)

394 [65]. Regardless of the algorithm used, ChIP-seq data provide a valuable resource to better

395 define the binding site sequences of transcription factors. Once identified, the location of motifs

396 within the peak region can be measured using CentriMo [66]. The ease of binning ChIP-seq data

397 and identifying motifs can allow for interesting biological comparisons, such as potential

398 differences in motifs due to differential enrichment levels under various experimental conditions.

399 It is important to note that, like all computational algorithms, motif-finding algorithms will

400 always return results, even if those results are not biologically significant. Care must be taken to

401 use the statistical results (E-value in MEME) to evaluate the results of these algorithms.

402 6.3 Searching Motifs Throughout the Genome and Within Peaks 
404 the occurrence of the motif to more accurately define the location of the TF binding site. This is

405 done using a position weight matrix (PWM) that describes the frequency distribution of

406 nucleotides at each position of the binding motif and is a common output of the aforementioned

407 motif finders. In some cases, multiple binding sites may occur within a single enriched region.

408 One significant challenge is the stringency of the cutoff to use in motif searches. Stringency

409 parameters can be verified by coupling the results of the search with true binding sites identified

410 from DNase I Footprinting or Electrophoretic Mobility Shift Assays (EMSA) experiments.

411 Further, the motif can be used to search the genome to identify potential binding sites

412 missed by ChIP-seq analysis. Although motif searches have a high false positive rate, we have

413 found that this analysis can provide important information regarding false negatives in the ChIP-

414 seq data, that is locations with a high value motif but lacking a corresponding ChIP-seq peak [2].

415 Such locations have been shown to be predicted binding sites of TFs that are blocked by the

416 binding of other proteins, such as nucleoid proteins H-NS [2]. There are many algorithms that

417 can search a genome for motif binding, including MAST [67], PatSer [68], and the Delila suite

418 [69]. We find the Delila software suite quite useful for fast, easily tunable genomic searches with

419 motifs. A particularly useful and unique feature of the Delila suite is the ability to visualize

420 graphically how a particular sequence was evaluated by a PWM using sequence walkers [69].

\section{Section 7: Correlation of ChIP-seq Data with Expression Data}

\subsection{Identification of Direct and Indirect Regulons in Bacteria}

423 Because ChIP-seq data provides information about where transcription factors bind in

424 vivo, correlation with expression data is crucial to identify regulons in bacteria. The source of

425 expression data can be either from whole genome microarray analyses, RNA-seq analyses, or a 
426 combination of both. Many studies have demonstrated that correlation of a global transcription

427 factor DNA binding with expression profiles of cells lacking that same TF compared to wild-

428 type cells reveals important information about the regulon of the transcription factor

$429[2,3,5,7,8,13,16,21,22,25]$. Importantly, these studies allow for the global identification of the

430 direct regulon - those operons that show a change in expression when the transcription factor is

431 deleted and also have a corresponding transcription factor ChIP-seq peak upstream - and the

432 indirect regulon - those operons that show a change in expression when the transcription factor is

433 deleted but do not have a corresponding transcription factor ChIP-seq peak upstream. Correlation

434 of ChIP-seq data with expression data also reveals the number of operons activated and repressed

435 by the TF on a global scale. Along with other data, this type of analysis can reveal important

436 features of transcription regulation and how bacteria respond to different environmental stimuli.

$437 \quad 7.2$ Visualizing Regulon Data on Metabolic Pathways

438 Once ChIP-seq and expression data are correlated, visualization of the data on metabolic

439 pathways is a useful strategy to gain a physiological understanding of the TF's function. Most

440 visualization tools are gene centric, but can be used with ChIP-seq data once peaks are associated

441 with genes. In a typical example, ChIP-seq occupancy and expression data are associated with

442 each gene in the pathway [2]. The pathway tools function of MetaCyc (www.metacyc.org) and

443 the Kyoto Encyclopedia of Genes and Genomes (KEGG; http://www.genome.jp/kegg/) are

444 excellent tools for an initial genomic overview of the data [70-72]. Both MetaCyc and KEGG

445 contain databases of gene and pathway information for many species and allow the simultaneous

446 mapping of -omics data to annotated metabolic reactions within the cell. For E. coli there are

447 specific on-line databases from which gene and pathway information can be obtained including

448 EcoCyc [73], PortEco [74], and EcoGene [75]. Additionally, the metabolic pathways can be 
449 downloaded from the user-generated site WikiPathways [76]. To generate custom metabolic

450 pathways, ProMetRa [77], GenMapp [78], or PathVisio [79] are good choices. However, it is

451 important to ensure proper representation of the pathway and any specific changes that exist in

452 the bacterial species being examined.

$453 \quad 7.3$ Validation of Regulon Analysis

454 While validation of all components of the direct and indirect regulon might not yet be

455 realistic, it is valuable to complement the conclusions of regulon analysis using in vitro or in vivo

456 methods. There are a variety of methods that can be used and here we will describe some

457 common examples. Any well-established in vitro DNA binding assay (e.g. EMSA, DNase I

458 footprinting, fluorescence polarization) can, in principle, be used to support TF binding to a

459 specific sequence of DNA that was enriched in ChIP-seq experiments. DNAse I footprinting is

460 advantageous because it determines the boundaries of the transcription factor binding site and is,

461 therefore, very informative for evaluating or optimizing binding motif analysis [3]. However it is

462 important to mention that the failure to observe binding in vitro does not rule out binding in vivo,

463 and could signify an interesting regulatory mechanism. For example, some binding events

464 require cooperative interactions with other TFs $[2,80]$ and/or may require specific cellular

465 conditions that are difficult to replicate in vitro.

466 To support direct transcriptional regulation, in vitro transcription assays or in vivo

467 measurements of reporter gene fusions to the promoter of interest (e.g. ß-galactosidase activity

468 assays or green fluorescent protein) or RNA levels by qRT-PCR can be performed with wild-

469 type strains and strains that contain deletions or mutations of various TFs. These assays can be

470 particularly informative for evaluating the effects of various growth conditions on transcriptional

471 regulation of a particular operon. In nearly every regulon analysis study to date, a significant 
472 number of ChIP-seq binding sites are located upstream of genes that fail to exhibit differential

473 expression under the growth conditions used in transcriptomic studies, leaving the biological

474 function of the binding site unknown. In some cases, alternative growth conditions or the

475 absence/inactivation of another TF may be required to observe an effect [2,3].

\section{Section 8: Summary and Perspectives}

477 Here, we have described an overview of obtaining and analyzing ChIP-seq data. ChIP-

478 seq analysis in bacteria is a powerful method of identifying in vivo binding sites of transcription

479 factors and their regulons. To date, several studies have combined ChIP-seq data and expression

480 data to gain a deep understanding of the regulon of transcription factors in multiple bacterial

481 species grown under a small number of growth conditions. For example, we can combine data

482 from our lab studying the oxygen or iron responsive TFs FNR, ArcA, IscR, and Fur to better

483 define the regulation of oxygen-dependent gene expression in E. coli (Figure 3). As the cost of

484 high-throughput sequencing decreases and more researchers have access to the technology, we

485 are hopeful that more $\mathrm{TF}$ regulons will be defined in bacteria, providing new and exciting

486 insights into bacterial regulons in a variety of species and growth conditions.

487 Acknowledgements

488 This work was funded by a grant from the NIH to PJK (GM045844). NAB was supported by the 489 UW-Madison NIH Chemistry Biology Interface Training Grant (T32GM008505). This work was 490 also funded in part by the DOE Great Lakes Bioenergy Research Center (DOE Office of Science 491 BER DE-FC02-07ER64494). 
493 Table 1. File formats used in ChIP-seq analysis.

494

\begin{tabular}{|c|c|c|}
\hline File Type & Brief Description & Use in Analysis \\
\hline FASTQ & $\begin{array}{l}\text { Illumina sequencing file } \\
\text { from experimental run }\end{array}$ & Raw ChIP-seq Data \\
\hline FASTQC & $\begin{array}{l}\text { Illumina quality control file } \\
\text { for each Illumina } \\
\text { sequencing run }\end{array}$ & $\begin{array}{l}\text { Evaluating ChIP-seq } \\
\text { Sequencing Data }\end{array}$ \\
\hline SAM & $\begin{array}{l}\text { Alignment file from } \\
\text { Bowtie2 or BWA }\end{array}$ & Aligned ChIP-seq File \\
\hline BAM & Binary SAM file & Aligned ChIP-seq File \\
\hline wiggle (WIG) & $\begin{array}{l}\text { File containing results of } \\
\text { enumerating read hits at } \\
\text { each base location }\end{array}$ & Visualization File \\
\hline ELAND & $\begin{array}{l}\text { Another alignment file } \\
\text { format, used as input in } \\
\text { MOSAiCS }\end{array}$ & For Peak Calling \\
\hline
\end{tabular}

495

496 

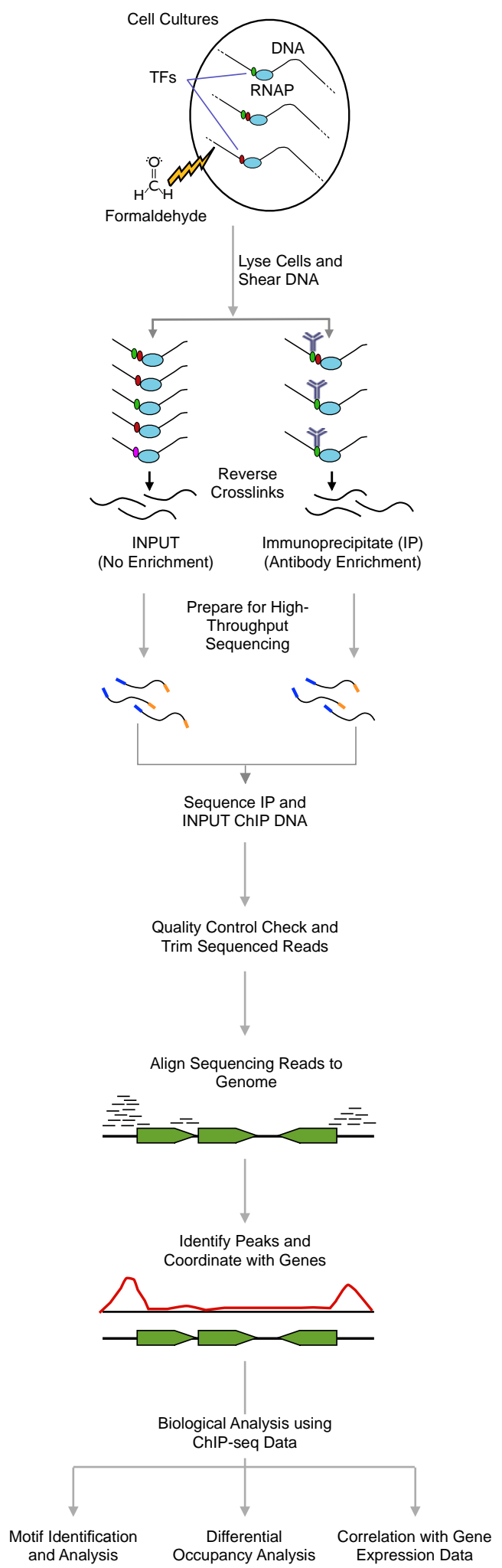
500 ChIP-seq begins with cell cultures grown in defined conditions. When cultures reach the desired 501 growth stage, they are treated with formaldehyde to crosslink proteins and DNA. The cells are 502 lysed and sheared so the average DNA length is $\sim 500 \mathrm{bp}$. The DNA-protein samples are split 503 into two populations, the Immunoprecipitate (IP) fraction, which is enriched for a particular 504 protein of interest using an antibody, and the Input fraction that is not enriched for any particular 505 protein of interest. The crosslinks are reversed by heat treatment and the DNA fragments are 506 subjected to high-throughput sequencing. After sequencing, the resulting sequencing reads are

507 examined for quality and trimmed based on read quality. The trimmed reads are then aligned to a 508 reference genome and algorithms and/or visual inspection identify ChIP-seq peaks. After peaks 509 are associated with genes downstream, several bioinformatic analyses can be performed 510 including motif identification and analysis, differential occupancy analysis, and correlation with 


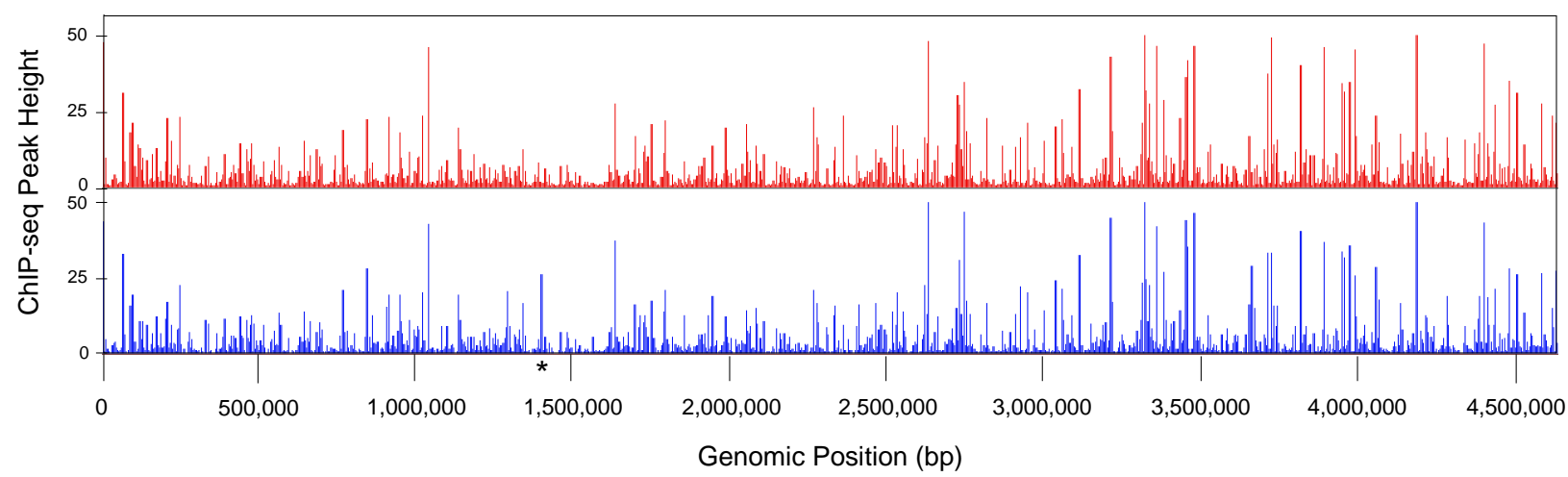

514

515

516

517

518 Shown are RNAP $\left(\sigma^{70}\right)$ ChIP-seq data traces collected from cultures grown under aerobic (red)

519 or anaerobic (blue) conditions [2]. ChIP-seq IP/INPUT ratio is shown on the y-axis and genomic

520 position is shown on the $\mathrm{x}$-axis. The asterisk indicates an example of differential binding

521 between growth conditions. This figure was generated in the MochiView browser [53].

522

523 
Figure 3. Example of combining bacterial regulons to better understand gene regulation in response to oxygen.

Shown is the proposed regulatory role of FNR (red ovals) [2], ArcA (purple square) [3], IscR (blue ovals) (Unpublished Data), and Fur (green rectangle) (Unpublished Data) on operons (blue, yellow, and gray circles) with a significant change in expression based on the presence or absence of $\mathrm{O}_{2}$. Lines indicate a regulatory role of a TF on expression from ChIP-chip/ChIP-seq and RNA expression data, either direct (blue circles) or indirect (yellow circles). Approximately $60 \%$ of the operons with an $\mathrm{O}_{2}$-dependent change in expression are regulated by FNR, ArcA, IscR, or Fur. The remaining $40 \%$ (gray circles) are regulated by other mechanisms not currently understood. 
[1] D.F. Browning, S.J. Busby, The regulation of bacterial transcription initiation, Nat Rev

[2] K.S. Myers, H. Yan, I.M. Ong, D. Chung, K. Liang, F. Tran, et al., Genome-scale analysis of Escherichia coli FNR reveals complex features of transcription factor binding, PLoS Genetics. 9 (2013) e1003565. doi:10.1371/journal.pgen.1003565.

[3] D.M. Park, M.S. Akhtar, A.Z. Ansari, R. Landick, P.J. Kiley, The bacterial response regulator ArcA uses a diverse binding site architecture to regulate carbon oxidation globally, PLoS Genetics. 9 (2013) e1003839. doi:10.1371/journal.pgen.1003839.

[4] J.R.J. Haycocks, P. Sharma, A.M. Stringer, J.T. Wade, D.C. Grainger, The molecular basis for control of ETEC enterotoxin expression in response to environment and host, PLoS Pathog. 11 (2015) e1004605. doi:10.1371/journal.ppat.1004605.

[5] C. Kahramanoglou, A.S.N. Seshasayee, A.I. Prieto, D. Ibberson, S. Schmidt, J. Zimmermann, et al., Direct and indirect effects of H-NS and Fis on global gene expression control in Escherichia coli, Nucleic Acids Res. 39 (2011) 2073-2091.

[6] S.S. Singh, N. Singh, R.P. Bonocora, D.M. Fitzgerald, J.T. Wade, D.C. Grainger, Widespread suppression of intragenic transcription initiation by H-NS, Genes Dev. 28 (2014) 214-219. doi:10.1101/gad.234336.113.

[7] A.I. Prieto, C. Kahramanoglou, R.M. Ali, G.M. Fraser, A.S.N. Seshasayee, N.M. Luscombe, Genomic analysis of DNA binding and gene regulation by homologous nucleoid-associated proteins IHF and HU in Escherichia coli K12, Nucleic Acids Res. 109 (2012) 3524-3537.

[8] D.R. Brown, G. Barton, Z. Pan, M. Buck, S. Wigneshweraraj, Nitrogen stress response and stringent response are coupled in Escherichia coli, Nat Comms. 5 (2014) 4115. doi: $10.1038 /$ ncomms5115.

[9] A. Fioravanti, C. Fumeaux, S.S. Mohapatra, C. Bompard, M. Brilli, A. Frandi, et al., DNA binding of the cell cycle transcriptional regulator GcrA depends on N6-adenosine methylation in Caulobacter crescentus and other Alphaproteobacteria, PLoS Genetics. 9 (2013) e1003541. doi:10.1371/journal.pgen.1003541.

[10] C. Fumeaux, S.K. Radhakrishnan, S. Ardissone, L. Théraulaz, A. Frandi, D. Martins, et al., Cell cycle transition from S-phase to G1 in Caulobacter is mediated by ancestral virulence regulators, Nat Comms. 5 (2014) 4081. doi:10.1038/ncomms5081.

[11] L. Solans, J. Gonzalo-Asensio, C. Sala, A. Benjak, S. Uplekar, J. Rougemont, et al., The PhoP-dependent ncRNA Mcr7 modulates the TAT secretion system in Mycobacterium tuberculosis, PLoS Pathog. 10 (2014) e1004183. doi:10.1371/journal.ppat.1004183.

[12] T.T. Perkins, M.R. Davies, E.J. Klemm, G. Rowley, T. Wileman, K. James, et al., ChIPseq and transcriptome analysis of the OmpR regulon of Salmonella enterica serovars Typhi and Typhimurium reveals accessory genes implicated in host colonization, Mol Microbiol. 87 (2013) 526-538. doi:10.1111/mmi.12111.

[13] C.J. Jones, D. Newsom, B. Kelly, Y. Irie, L.K. Jennings, B. Xu, et al., ChIP-Seq and RNA-Seq reveal an AmrZ-mediated mechanism for cyclic di-GMP synthesis and biofilm development by Pseudomonas aeruginosa, PLoS Pathog. 10 (2014) e1003984. doi:10.1371/journal.ppat.1003984.

[14] S. Imam, D.R. Noguera, T.J. Donohue, Global analysis of photosynthesis transcriptional regulatory networks, PLoS Genetics. 10 (2014) e1004837. 
doi:10.1371/journal.pgen.1004837.

[15] J.C. Crack, J. Munnoch, E.L. Dodd, F. Knowles, M.M. Al Bassam, S. Kamali, et al., NsrR from Streptomyces coelicolor is a Nitric Oxide-Sensing [4Fe-4S] Cluster Protein with a Specialized Regulatory Function, J Biol Chem. (2015). doi:10.1074/jbc.M115.643072.

[16] B.W. Davies, R.W. Bogard, J.J. Mekalanos, Mapping the regulon of Vibrio cholerae ferric uptake regulator expands its known network of gene regulation, Proc Natl Acad Sci USA. (2011).

[17] T.G. Dong, J.J. Mekalanos, Characterization of the RpoN regulon reveals differential regulation of T6SS and new flagellar operons in Vibrio cholerae O37 strain V52, Nucleic Acids Res. 40 (2012) 7766-7775. doi:10.1093/nar/gks567.

[18] J.C. van Kessel, L.E. Ulrich, I.B. Zhulin, B.L. Bassler, Analysis of activator and repressor functions reveals the requirements for transcriptional control by LuxR, the master regulator of quorum sensing in Vibrio harveyi, mBio. 4 (2013). doi:10.1128/mBio.00378-13.

[19] B. Blasco, J.M. Chen, R. Hartkoorn, C. Sala, S. Uplekar, J. Rougemont, et al., Virulence regulator EspR of Mycobacterium tuberculosis is a nucleoid-associated protein, PLoS Pathog. 8 (2012) e1002621. doi:10.1371/journal.ppat.1002621.

[20] D.S. Lun, A. Sherrid, B. Weiner, D.R. Sherman, J.E. Galagan, A blind deconvolution approach to high-resolution mapping of transcription factor binding sites from ChIP-seq data, Genome Biol. 10 (2009) R142. doi:10.1186/gb-2009-10-12-r142.

[21] K.J. Minch, T.R. Rustad, E.J.R. Peterson, J. Winkler, D.J. Reiss, S. Ma, et al., The DNA-binding network of Mycobacterium tuberculosis, Nat Comms. 6 (2015) 5829-. doi: $10.1038 /$ ncomms6829.

[22] J.E. Galagan, K. Minch, M. Peterson, A. Lyubetskaya, E. Azizi, L. Sweet, et al., The Mycobacterium tuberculosis regulatory network and hypoxia, Nature. 499 (2013) 178183. doi:10.1038/nature12337.

[23] D.M. Fitzgerald, R.P. Bonocora, J.T. Wade, Comprehensive mapping of the Escherichia coli flagellar regulatory network, PLoS Genetics. 10 (2014) e1004649. doi:10.1371/journal.pgen.1004649.

[24] B.L. Petrone, A.M. Stringer, J.T. Wade, Identification of HilD-regulated genes in Salmonella enterica serovar Typhimurium, J Bacteriol. 196 (2014) 1094-1101. doi:10.1128/JB.01449-13.

[25] A.M. Stringer, S. Currenti, R.P. Bonocora, C. Baranowski, B.L. Petrone, M.J. Palumbo, et al., Genome-scale analyses of Escherichia coli and Salmonella enterica AraC reveal noncanonical targets and an expanded core regulon, J Bacteriol. 196 (2014) 660-671. doi:10.1128/JB.01007-13.

[26] S. Cho, Y.B. Cho, T.J. Kang, S.C. Kim, B. Palsson, B.K. Cho, The architecture of ArgRDNA complexes at the genome-scale in Escherichia coli, Nucleic Acids Res. 43 (2015) 3079-3088. doi:10.1093/nar/gkv150.

[27] N. Carraro, D. Matteau, P. Luo, S. Rodrigue, V. Burrus, The master activator of IncA/C conjugative plasmids stimulates genomic islands and multidrug resistance dissemination, PLoS Genetics. 10 (2014) e1004714. doi:10.1371/journal.pgen.1004714.

[28] D. Poulin-Laprade, D. Matteau, P.-É. Jacques, S. Rodrigue, V. Burrus, Transfer activation of SXT/R391 integrative and conjugative elements: unraveling the SetCD regulon, Nucleic Acids Res. 43 (2015) 2045-2056. doi:10.1093/nar/gkv071. 
S.W. Seo, D. Kim, H. Latif, E.J. O'Brien, R. Szubin, B.Ø. Palsson, Deciphering Fur transcriptional regulatory network highlights its complex role beyond iron metabolism in Escherichia coli, Nat Comms. 5 (2014) 4910. doi:10.1038/ncomms5910. R.P. Bonocora, J.T. Wade, ChIP-Seq for Genome-Scale Analysis of Bacterial DNABinding Proteins, Methods Mol Biol. 1276 (2015) 327-340. doi:10.1007/978-1-49392392-2_20.

[31] J. Galagan, A. Lyubetskaya, A. Gomes, ChIP-Seq and the complexity of bacterial transcriptional regulation, Curr. Top. Microbiol. Immunol. 363 (2013) 43-68.

[32] S. Jaini, A. Lyubetskaya, A. Gomes, M. Peterson, S.T. Park, Transcription Factor Binding Site Mapping Using ChIP-Seq, (2014). doi:10.1128/microbiolspec.MGM20035-2013.

[33] S.E. Davis, R.A. Mooney, E.I. Kanin, J. Grass, R. Landick, A.Z. Ansari, Mapping E. coli RNA Polymerase and associated transcription factors and identifying promoters genome-wide, Meth Enzymol. 498 (2011) 449-471.

[34] D. Thieffry, A.M. Huerta, E. Pérez-Rueda, J. Collado-Vides, From specific gene regulation to genomic networks: a global analysis of transcriptional regulation in Escherichia coli, Bioessays. 20 (1998) 433-440. doi:10.1002/(SICI)15211878(199805)20:5<433::AID-BIES10>3.0.CO;2-2.

K. Liang, S. Keleş, Detecting differential binding of transcription factors with ChIP-seq, Bioinformatics. 28 (2012) 121-122.

$677 \quad[46]$ K. Liang, S. Keleş, Normalization of ChIP-seq data with control, BMC Bioinformatics. 13 (2012) 199.

P.J. Park, ChIP-seq: advantages and challenges of a maturing technology, Nat Rev Genet. 10 (2009) 669-680.

[40] B. Langmead, S.L. Salzberg, Fast gapped-read alignment with Bowtie 2, Nat Methods. 9 (2012) 357-359.

[41] H. Li, R. Durbin, Fast and accurate short read alignment with Burrows-Wheeler transform, Bioinformatics. 25 (2009) 1754-1760.

[42] H. Li, B. Handsaker, A. Wysoker, T. Fennell, J. Ruan, N. Homer, et al., The Sequence Alignment/Map format and SAMtools, Bioinformatics. 25 (2009) 2078-2079. doi:10.1093/bioinformatics/btp352.

[43] H. Ji, H. Jiang, W. Ma, D.S. Johnson, R.M. Myers, W.H. Wong, An integrated software system for analyzing ChIP-chip and ChIP-seq data, Nat Biotechnol. 26 (2008) 12931300.

[44] Y. Zhang, T. Liu, C. Meyer, J. Eeckhoute, D. Johnson, B. Bernstein, et al., Model-based analysis of ChIP-Seq (MACS), Genome Biol. 9 (2008) R137.

[45] D. Chung, P.F. Kuan, S. Keleş, Analysis of ChIP-seq Data with "mosaics" Package, (2012).

[46] D. Chung, D. Park, K. Myers, J. Grass, P. Kiley, R. Landick, et al., dPeak: High 
resolution identification of transcription factor binding sites from PET and SET ChIPSeq data, PLoS Comput Biol. 9 (2013) e1003246.

[47] A.L.C. Gomes, T. Abeel, M. Peterson, E. Azizi, A. Lyubetskaya, L. Carvalho, et al., Decoding ChIP-seq with a double-binding signal refines binding peaks to singlenucleotides and predicts cooperative interaction, Genome Res. 24 (2014) 1686-1697. doi:10.1101/gr.161711.113.

[48] Y. Guo, S. Mahony, D.K. Gifford, High resolution genome wide binding event finding and motif discovery reveals transcription factor spatial binding constraints, PLoS Comput Biol. 8 (2012) e1002638. doi:10.1371/journal.pcbi.1002638.

[49] X. Zhang, G. Robertson, M. Krzywinski, K. Ning, A. Droit, S. Jones, et al., PICS: Probabilistic Inference for ChIP-seq, Biometrics. 67 (2010) 151-163. doi:10.1111/j.1541-0420.2010.01441.x.

[50] N. Bonhoure, G. Bounova, D. Bernasconi, V. Praz, F. Lammers, D. Canella, et al., Quantifying ChIP-seq data: a spiking method providing an internal reference for sampleto-sample normalization, Genome Res. 24 (2014) 1157-1168. doi:10.1101/gr.168260.113.

[51] A. Valouev, D.S. Johnson, A. Sundquist, C. Medina, E. Anton, S. Batzoglou, et al., Genome-wide analysis of transcription factor binding sites based on ChIP-seq data, Nat Methods. 5 (2008) 829-834.

[52] E. Wilbanks, M. Facciotti, Evaluation of algorithm performance in ChIP-seq peak detection, PLoS ONE. (2010).

[53] O.R. Homann, A.D. Johnson, MochiView: versatile software for genome browsing and DNA motif analysis, BMC Biol. 8 (2010) 49.

[54] W.J. Kent, C.W. Sugnet, T.S. Furey, K.M. Roskin, T.H. Pringle, A.M. Zahler, et al., The human genome browser at UCSC, Genome Res. 12 (2002) 996-1006. doi:10.1101/gr.229102.

[55] L.D. Stein, C. Mungall, S. Shu, M. Caudy, M. Mangone, A. Day, et al., The generic genome browser: a building block for a model organism system database, Genome Res. 12 (2002) 1599-1610. doi:10.1101/gr.403602.

[56] M.E. Skinner, A.V. Uzilov, L.D. Stein, C.J. Mungall, I.H. Holmes, JBrowse: a nextgeneration genome browser, Genome Res. 19 (2009) 1630-1638. doi:10.1101/gr.094607.109.

[57] D. Kim, J.S.-J. Hong, Y. Qiu, H. Nagarajan, J.-H. Seo, B.-K. Cho, et al., Comparative analysis of regulatory elements between Escherichia coli and Klebsiella pneumoniae by genome-wide transcription start site profiling, PLoS Genetics. 8 (2012) e1002867. doi:10.1371/journal.pgen.1002867.

[58] T. Conway, J.P. Creecy, S.M. Maddox, J.E. Grissom, T.L. Conkle, T.M. Shadid, et al., Unprecedented high-resolution view of bacterial operon architecture revealed by RNA sequencing, mBio. 5 (2014) e01442-14. doi:10.1128/mBio.01442-14.

[59] M.K. Das, H.-K. Dai, A survey of DNA motif finding algorithms, BMC Bioinformatics. 8 Suppl 7 (2007) S21. doi:10.1186/1471-2105-8-S7-S21.

[60] T.L. Bailey, N. Williams, C. Misleh, W.W. Li, MEME: discovering and analyzing DNA and protein sequence motifs, Nucleic Acids Res. 34 (2006) W369-73. doi:10.1093/nar/gkl198.

[61] P. Machanick, T.L. Bailey, MEME-ChIP: motif analysis of large DNA datasets, Bioinformatics. 27 (2011) 1696-1697. 
[62] T.L. Bailey, M. Boden, F.A. Buske, M. Frith, C.E. Grant, L. Clementi, et al., MEME SUITE: tools for motif discovery and searching, Nucleic Acids Res. 37 (2009) W202-8. doi:10.1093/nar/gkp335.

[63] M. Defrance, J. van Helden, info-gibbs: a motif discovery algorithm that directly optimizes information content during sampling, Bioinformatics. 25 (2009) 2715-2722. doi:10.1093/bioinformatics/btp490.

[64] G.Z. Hertz, G.W. Hartzell, G.D. Stormo, Identification of consensus patterns in unaligned DNA sequences known to be functionally related, Comput. Appl. Biosci. 6

732

733 (1990) 81-92.

[65] M. Thomas-Chollier, M. Defrance, A. Medina-Rivera, O. Sand, C. Herrmann, D. Thieffry, et al., RSAT 2011: regulatory sequence analysis tools, Nucleic Acids Res. 39 (2011) W86-91. doi:10.1093/nar/gkr377.

[66] T.L. Bailey, P. Machanick, Inferring direct DNA binding from ChIP-seq, Nucleic Acids Res. 40 (2012) e128. doi:10.1093/nar/gks433.

[67] S. Gupta, J.A. Stamatoyannopoulos, T.L. Bailey, W.S. Noble, Quantifying similarity between motifs, Genome Biol. 8 (2007) R24. doi:10.1186/gb-2007-8-2-r24.

[68] G.Z. Hertz, G.D. Stormo, Identifying DNA and protein patterns with statistically significant alignments of multiple sequences, Bioinformatics. 15 (1999) 563-577.

[69] T.D. Schneider, G.D. Stormo, M.A. Yarus, L. Gold, Delila system tools, Nucleic Acids Res. 12 (1984) 129-140.

[70] R. Caspi, T. Altman, R. Billington, K. Dreher, H. Foerster, C.A. Fulcher, et al., The MetaCyc database of metabolic pathways and enzymes and the BioCyc collection of Pathway/Genome Databases, Nucleic Acids Res. 42 (2014) D459-71. doi:10.1093/nar/gkt1103.

[71] M. Kanehisa, S. Goto, KEGG: Kyoto Encyclopedia of Genes and Genomes, Nucleic Acids Res. 28 (2000) 27-30. doi:10.1093/nar/28.1.27.

[72] M. Kanehisa, S. Goto, Y. Sato, M. Kawashima, M. Furumichi, M. Tanabe, Data, information, knowledge and principle: back to metabolism in KEGG, Nucleic Acids Res. 42 (2014) D199-205. doi:10.1093/nar/gkt1076.

[73] I.M. Keseler, J. Collado-Vides, A. Santos-Zavaleta, M. Peralta-Gil, S. Gama-Castro, L. Muñiz-Rascado, et al., EcoCyc: a comprehensive database of Escherichia coli biology, Nucleic Acids Res. 39 (2011) D583-90.

[74] J.C. Hu, G. Sherlock, D.A. Siegele, S.A. Aleksander, C.A. Ball, J. Demeter, et al., PortEco: a resource for exploring bacterial biology through high-throughput data and analysis tools, Nucleic Acids Res. 42 (2014) D677-84. doi:10.1093/nar/gkt1203.

[75] J. Zhou, K.E. Rudd, EcoGene 3.0, Nucleic Acids Res. 41 (2013) D613-24. doi:10.1093/nar/gks1235.

[76] A.R. Pico, T. Kelder, M.P. van Iersel, K. Hanspers, B.R. Conklin, C. Evelo, WikiPathways: pathway editing for the people, PLoS Biol. 6 (2008) e184. doi:10.1371/journal.pbio.0060184.

[77] H. Neuweger, M. Persicke, S.P. Albaum, T. Bekel, M. Dondrup, A.T. Hüser, et al., Visualizing post genomics data-sets on customized pathway maps by ProMeTra aeration-dependent gene expression and metabolism of Corynebacterium glutamicum as an example, BMC Syst Biol. 3 (2009) 82.

[78] K.D. Dahlquist, N. Salomonis, K. Vranizan, S.C. Lawlor, B.R. Conklin, GenMAPP, a new tool for viewing and analyzing microarray data on biological pathways, Nat Genet. 
31 (2002) 19-20. doi:10.1038/ng0502-19.

[79] M. Kutmon, M.P. van Iersel, A. Bohler, T. Kelder, N. Nunes, A.R. Pico, et al., PathVisio 3: an extendable pathway analysis toolbox, PLoS Comput Biol. 11 (2015) e1004085.

773 doi:10.1371/journal.pcbi.1004085.

774

[80] D.J. Lee, S.D. Minchin, S.J.W. Busby, Activating transcription in bacteria, Annu Rev

775 Microbiol. 66 (2012) 125-152. doi:10.1146/annurev-micro-092611-150012. 


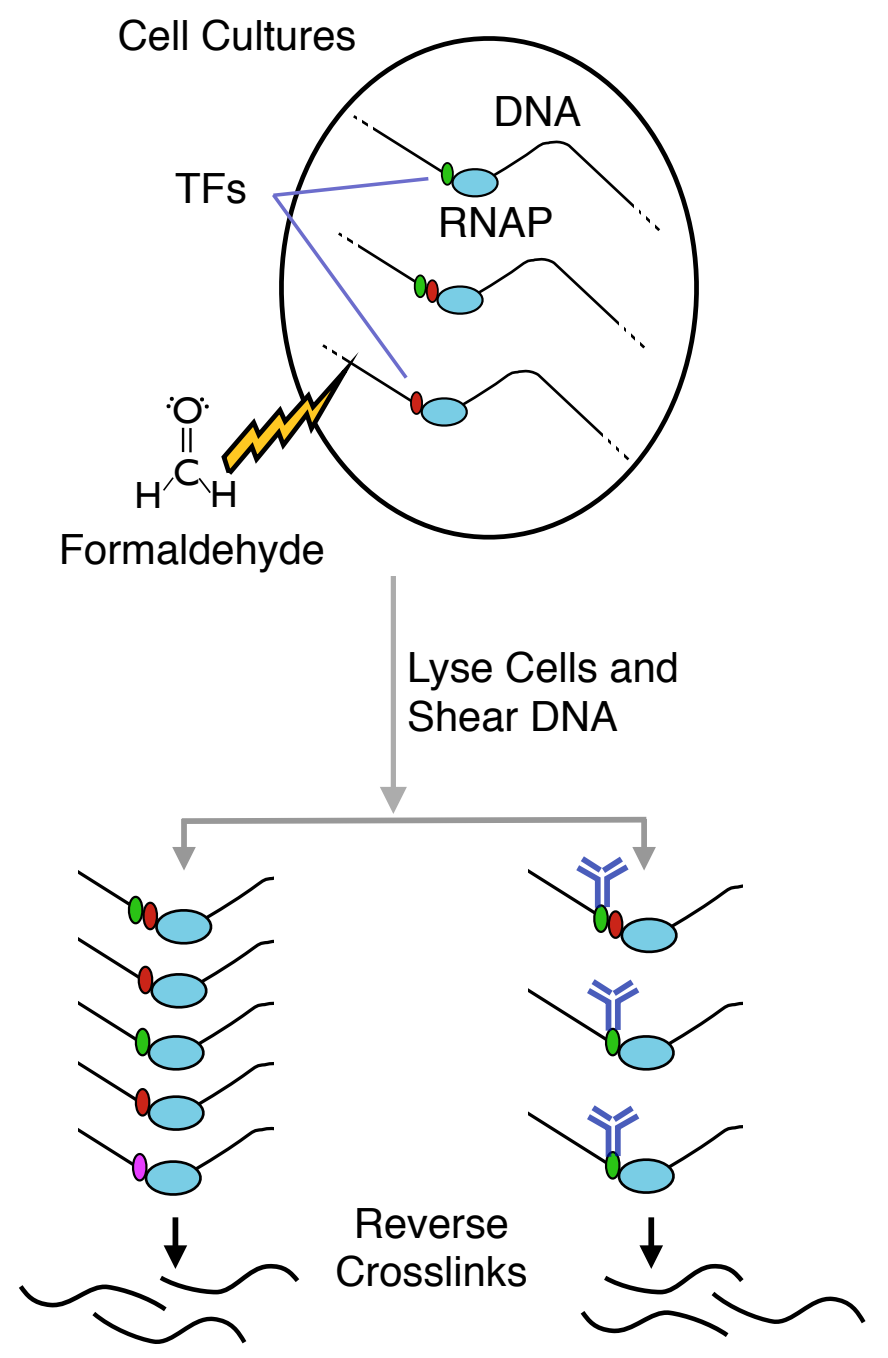

INPUT Immunoprecipitate (IP)

(No Enrichment) (Antibody Enrichment)

Prepare for High-

Throughput

Sequencing

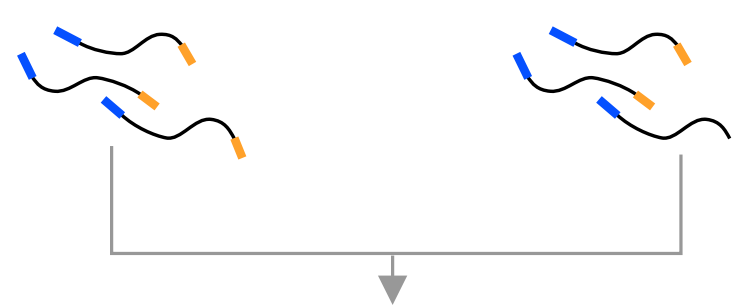

Sequence IP and

INPUT ChIP DNA

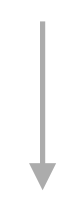

Quality Control Check and

Trim Sequenced Reads

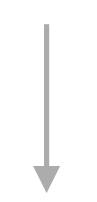

Align Sequencing Reads to

Genome

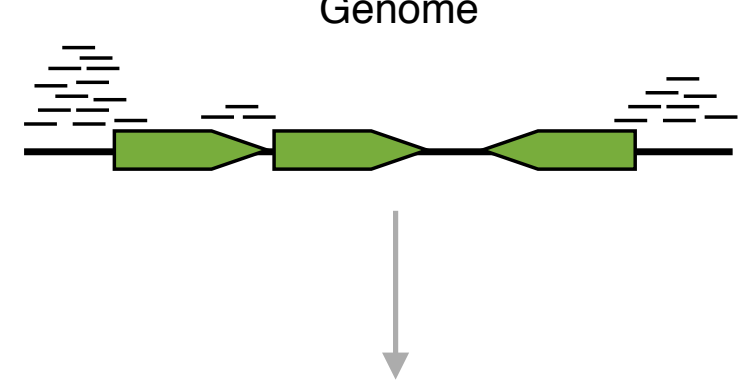

Identify Peaks and

Coordinate with Genes

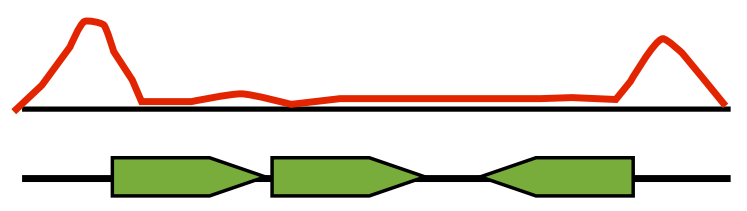

Biological Analysis using

ChIP-seq Data

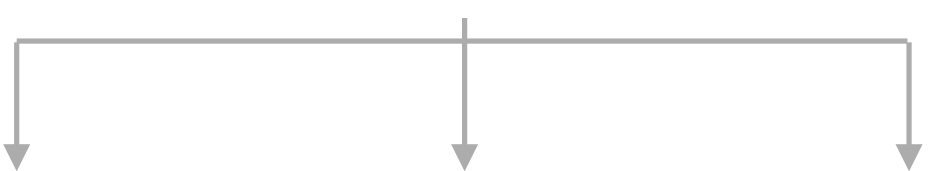

Motif Identification and Analysis 


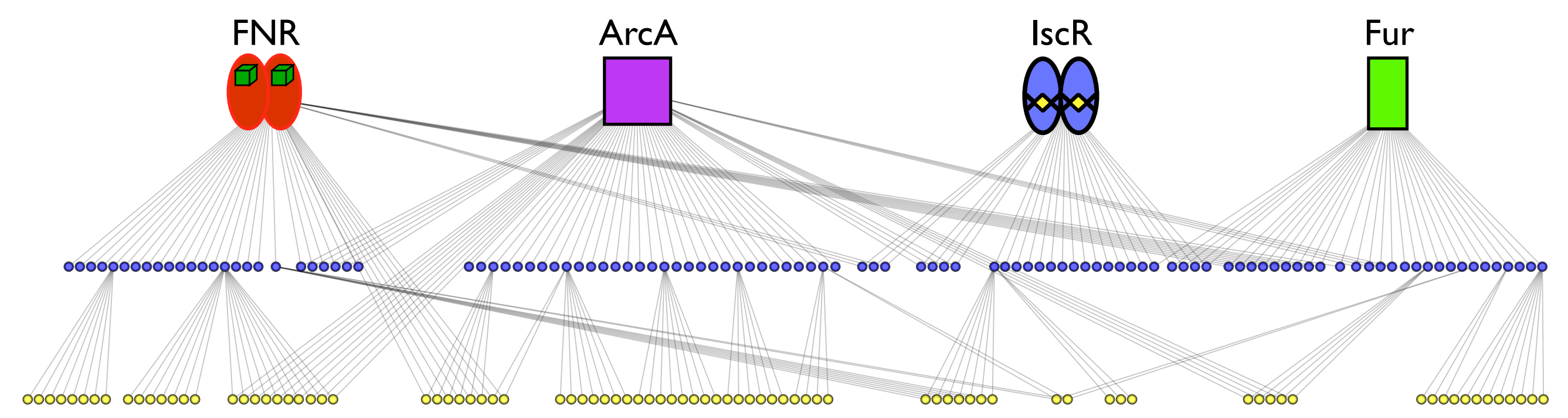

000000000000000000000000000000000000000000000000000000000000000000000000000 Unknown Regulatory Mechanism 
Table 1. File formats used in ChIP-seq analysis.

\begin{tabular}{|c|c|c|}
\hline File Type & Brief Description & Use in Analysis \\
\hline FASTQ & $\begin{array}{l}\text { Illumina sequencing file } \\
\text { from experimental run }\end{array}$ & Raw ChIP-seq Data \\
\hline FASTQC & $\begin{array}{l}\text { Illumina quality control file } \\
\text { for each Illumina } \\
\text { sequencing run }\end{array}$ & $\begin{array}{l}\text { Evaluating ChIP-seq } \\
\text { Sequencing Data }\end{array}$ \\
\hline SAM & $\begin{array}{l}\text { Alignment file from } \\
\text { Bowtie } 2 \text { or BWA }\end{array}$ & Aligned ChIP-seq File \\
\hline BAM & Binary SAM file & Aligned ChIP-seq File \\
\hline wiggle (WIG) & $\begin{array}{l}\text { File containing results of } \\
\text { enumerating read hits at } \\
\text { each base location }\end{array}$ & Visualization File \\
\hline ELAND & $\begin{array}{c}\text { Another alignment file } \\
\text { format, used as input in } \\
\text { MOSAiCS }\end{array}$ & For Peak Calling \\
\hline
\end{tabular}

